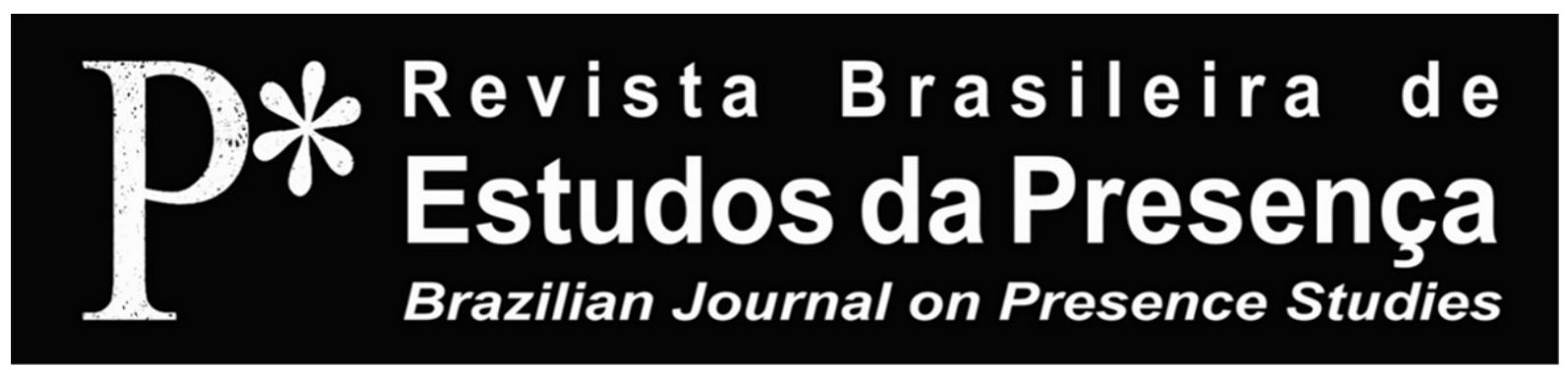

ISSN 2237-2660

\title{
L'Attention à travers le Mouvement: de la méthode Feldenkrais comme amorce d'une pensée de l'attention
}

\author{
Marie Bardet \\ Université Paris 8 - Paris, France
}

RÉSUMÉ - L'Attention à travers le Mouvement: de la méthode Feldenkrais comme amorce d'une pensée de l'attention - Le présent article propose de reprendre certains aspects théoriques et pratiques de la méthode Feldenkrais, en tant que cette méthode somatique annonce déplacer la perspective d'un travail corporel sur un corps-objet vers celle d'une éducation à certaine attention à travers le mouvement. L'étude philosophique d'un tel déplacement permet d'en discuter les effets conceptuels et concrets à partir d'une triple caractérisation de cette attention (awareness): dynamique, différentielle et tendancielle. Chacun de ces caractères est étudié tour à tour, à partir d'exemples concrets de la pratique, de citations de textes de Feldenkrais, et en revenant sur une référence fondatrice: la loi de Weber-Fechner.

Mots-clés: Attention. Sensation. Fechner. Feldenkrais. Philosophie.

ABSTRACT - Awareness through Movement: Feldenkrais method as a way of thinking awareness - Our proposal in this paper aims at clarifying some of the theoretical and practical assumptions of the Feldenkrais method, considering the change implied by his project of a somatic method that does not want to exercise a body-object, but much more to teach what he named awareness through movements. A philosophical study of such a change allows us to discuss its concrete and conceptual effects, from a triple characterization of this awareness: dynamic, differential, and made of tendencies. Each one of those three aspects is successively considered studying some examples of the lessons, some Moshe Feldenkrais writings and a historical reference: the Weber-Fechner law.

Keywords: Awareness. Sensation. Fechner. Feldenkrais. Philosophy.

RESUMO - A Atenção através do Movimento: o método Feldenkrais como disparador de um pensamento sobre a atenção - $\mathrm{O}$ presente artigo propóe retomar certos aspectos teóricos e práticos do método Feldenkrais, compreendendo-o como método somático que pretende deslocar a perspectiva do trabalho corporal sobre um corpo-objeto para a de uma educação centrada na atenção através do movimento. $\mathrm{O}$ estudo filosófico de tal deslocamento permite discutir seus efeitos conceituais e concretos a partir de uma tripla caracterização dessa atenção (awareness): dinâmica, diferencial e tendencial. Cada uma dessas características é estudada uma a uma, a partir de exemplos concretos da prática, de citações de textos de Feldenkrais, e retomando uma referência fundadora: a lei de Weber-Fechner. Palavras-chave: Atenção. Sensação. Fechner. Feldenkrais. Filosofia. 
Les recherches sur les somatiques (Hanna, 1995, p. 341) exigent des approches nécessairement pluridisciplinaires et repensant le rapport entre théorie et pratique. C'est dans cette direction que s'inscrit le travail du groupe de recherche Soma\&Po qui réunit, au sein du département de danse de l'Université Paris 8, des chercheuses provenant de différentes disciplines académiques (philosophie, biologie, écologie, études en danse, etc.) et de diverses pratiques (Feldenkrais, Body-Mind Centering ${ }^{\circledR}$, danse-improvisation, danse/nature, activisme queer etc.). Ce travail collectif vise à faire émerger une ou des épistémologies des méthodes somatiques à partir de dispositifs théoricopratiques qui interrogent, essentiellement, les portées et les limites de la question du politique dans les discours et pratiques somatiques. Le présent article, même si écrit individuellement, doit beaucoup à ce travail d'équipe.

En outre, ce texte s'inscrit plus largement dans une recherche en philosophie qui s'intéresse aux pratiques somatiques (en particulier à partir de ma propre expérience de la méthode Feldenkrais en tant qu'élève, depuis plus de dix ans) et à la pratique et la pensée de l'improvisation en danse. C'est dans ce cadre que surgit la question initiale de ce travail: dans quelle mesure, et au prix de quels déplacements pratiques et conceptuels, peut-on dire que les différentes méthodes somatiques (dont les plus connues sont Alexander, Feldenkrais, BMC, eutonie) peuvent être comprises comme des méthodes corporelles qui, paradoxalement, n'entrainent pas directement le corps? Et plus particulièrement ici, quels effets peut avoir ce déplacement lorsque Feldenkrais annonce travailler à une prise de conscience à travers le mouvement?

La question de ce changement de paradigme (effectif ou en tout cas annoncé) se pose conjointement pour nos regards, nos analyses et notre pensée, qui devraient alors moins porter sur le corps qu'ils ne chercheraient à s'exercer à travers le mouvement (de là les dispositifs nécessairement théorico-pratiques), ne pouvant pas considérer le corps comme un pur objet d'étude.

En effet, le défi de ces recherches collectives et singulières est donc double: d'un côté élaborer des épistémologies de ces pratiques somatiques au plus près de leur pensée/pratique, exigeant des processus de recherche mêlant lecture des textes, expérience concrètes, retour sur la pratique avec des praticiens et des élèves, et conversation entre différents champ de recherche. D'un autre côté, donner une 
certaine consistance à ce que ces expériences produisent comme concepts et peuvent déplacer dans une philosophie contemporaine - au sens de coprésente à des expériences actuelles. Encore une fois donc, la philosophie qui se frotte à ces pratiques ne se retrouve pas tant face à l'apparition du corps comme un nouvel objet d'étude, qu'elle n'est confrontée à l'exigence d'une pensée située, et à l'émergence de conceptions renouvelées, entre autres, de la sensation, de la conscience, de l'expérience.

Posons donc ici ces questions pour le cas particulier de la méthode Feldenkrais, en prenant nécessairement en compte à la fois l'expérience des cours collectifs et la lecture de ses textes. Si cette méthode ne travaille pas sur le corps, que fait-elle ou prétend-elle faire? Et en quoi cela peut-il interroger nos concepts habituels de conscience, et nourrir une pensée philosophique occidentale? La méthode fondée par Moshe Feldenkrais (1904-1984) s'est répandue à travers la transmission qu'en a fait Feldenkrais lui-même, puis les personnes qui lui ont succédé et organisent depuis lors les formations de praticiens dans de nombreux pays. Les praticiens Feldenkrais enseignent la méthode à ce qu'ils appellent souvent des élèves - et non des patients, ce qui peut dénoter l'intention d'une certaine orientation vers un apprentissage plutôt qu'une cure - sous forme de cours qui peuvent être individuels ou collectifs. Feldenkrais va plus particulièrement nommer les séances collectives awareness through movement, habituellement traduit en français par l'expression: prise de conscience par le mouvement. Pourtant, awareness n'est pas consciousness, et l'on peut préférer le terme d'attention pour traduire ce terme. Reste à voir quels seraient quelques-uns des déplacements conceptuels et pratiques d'un travail non pas sur le corps, mais par le mouvement, développant une attention à travers le mouvement plutôt qu'une conscience de quelque chose, du corps.

\section{Une Relation plus qu'une Chose}

Si l'on peut dire que la méthode Feldenkrais ne travaille pas sur le corps, c'est tout d'abord dans le sens où Feldenkrais annonce que la méthode opère sur des relations plus que sur des choses. En effet, il ne dit pas qu'il travaille sur le corps, comme un objet unique, défini, séparé, mais sur une relation multiple et plus large: entre «[...] le squelette, les muscles, le système nerveux et l'environnement» (Feldenkrais, 2010b, p. 59). Il revient également sur cette idée dans 
un autre texte écrit dans la même année 1979, avec une légère variation dans les termes (c'est d'ailleurs une caractéristique des textes de Feldenkrais comme de nombreux textes théoriques de fondateurs de méthodes somatiques: la grande variabilité dans les termes utilisés rendant le corpus peu systématique et systématisable):

[...] nous devons considérer les êtres humains dans leur intégralité. Une personne est composée de trois entités: le système nerveux, qui est le noyau; le corps - squelette, viscères et muscles - qui est l'enveloppe; et l'environnement, que sont l'espace, la gravité et la société ${ }^{2}$ (Feldenkrais, 2010a, p. 32).

Feldenkrais annonce donc qu'il entend prendre en considération l'humain tout entier, ou plus précisément encore comme une relation entre différents éléments. Cette relation est profondément dynamique, changeante, parce qu'elle est variation entre les différents éléments. Alors, si le travail de la méthode Feldenkrais annonce que ces cours collectifs travaillent à une attention à travers le mouvement, c'est une attention à une relation variable entre plusieurs éléments plutôt qu'une prise de conscience d'un corps-objet-physique. Et si apprentissage il y a, c'est de l'expérience de «variabilité» possible, à partir de ces petites variations (Bardet; Ginot, 2012, p. 22-23). Cela exige donc de concevoir, du point de vue de la philosophie, une attention dynamique et différentielle, qui se distingue sur ce point déjà d'une conscience de quelque chose. Afin d'en considérer les soubassements, les déplacements et les limites, aussi bien concrets que conceptuels, je partirai de la situation de tout début de séance collective: le scanning, en la faisant dialoguer avec des textes de Moshe Feldenkrais, essentiellement Mind and Body (Feldenkrais, 2010a).

\section{Une Attention Dynamique}

Allongez-vous sur le sol. Quelle sensation globale avez-vous? Quelles sont les parties en contact avec le sol? Par exemple, de quelle manière votre pied est-il en contact avec le sol? Quelle est la zone de contact? Qu'est-ce que vous sentez de ce contact? Avec quelle qualité touche-t-elle le sol?

Quelle est la partie de votre bassin en contact? Y a-t-il un côté du bassin qui touche plus que l'autre? Et quelle est la qualité du contact?

Le scanning de début de séance collective, qui sera repris à différents moments de la séance, chercherait autant à clarifier l'image de certaines parties qu'à porter son attention sur des manières d'être 
en relation avec le sol, de toucher, mais surtout à se rendre attentif aux changements qui auront lieu au cours de la séance. Ainsi la phrase "[...] la posture n'est pas un état statique mais une activité dynamique» (Feldenkrais, 2010a, p. 42) le dit bien: la posture est toujours prise dans une relation entre plusieurs éléments constitutifs de la personne, et une relation essentiellement dynamique, et donc changeante. L'attention portée à la posture ne peut pas être de la posture, mais doit être l'attention d'une relation, et se déployer à travers une relation.

L'attention recherchée est donc essentiellement une attention dynamique et portée sur les petits changements. Tel semble être en tous cas le sens de ce scanning:

Je commence en demandant aux gens de s'allonger sur le dos (selon le même principe de réduction de la gravité) et d'apprendre à s'observer [to scan] eux-mêmes. C'est-à-dire qu'ils examinent attentivement le contact de leurs corps avec le sol et apprennent petit à petit à apercevoir des différences remarquables - des points où le contact est faible ou inexistant et d'autres où il est plein et distinct ${ }^{3}$ (Feldenkrais, 2010b, p. 76).

Ce scanning de départ, qui entend non pas dire comment le corps doit reposer sur le sol, mais donner un élément d'auto-apprentissage, va se répéter au cours de la séance, et établir ainsi des jalons subjectifs pour affiner la sensation de la différence. C'est ainsi que l'on peut comprendre que Feldenkrais va mettre le focus perceptif sur les différences: à commencer par les différences dans l'orientation et la réaction gravitaire, entre un côté et l'autre, avant/après etc.:

Le scanning mental consiste à écouter et devenir attentif à la différence de sensation dans la mémoire motrice des muscles de chaque côté, et à la sensation de changement d'orientation dans l'espace ${ }^{4}$ (Feldenkrais, 2010a, p. 39).

Mais il semblerait que cette sensation de la différence n'est pas seulement comparative (entre un côté et l'autre, un moment et un autre), elle est elle-même différentielle: «[...] sensibilité, - c'est-à-dire, la capacité de sentir la différence ${ }^{5}$ [...]»(Feldenkrais, 2010a, p. 37). C'est ainsi qu'au cour du travail de scanning, et tout au long de la séance, la méthode part d'un principe: non seulement l'attention est portée sur une différence comparative, mais elle fait appel à une sensibilité fondamentalement différentielle. 


\section{Une Sensibilité Différentielle}

En effet, cette affirmation d'une sensibilité essentiellement différentielle s'appuie sur la référence faite, dans le même texte, à la loi de Weber-Fechner:

La réduction progressive de tout effort inutile est nécessaire afin d'augmenter la sensibilité kinesthésique, sans laquelle une personne ne peut pas s'autoréguler. La loi de Fechner-Weber montre clairement que c'est le cas. Cette loi établit que pour une grande partie des sensations et activités humaines, la différence du stimulus produisant la plus petite différence perceptible dans la sensation correspond toujours à la même proportion de tout le stimulus ${ }^{6}$ (Feldenkrais, 2010b, p. 37).

Tant Feldenkrais que d'autres fondateurs de méthodes somatiques font souvent référence à cette loi de la première psychophysique (XIX ${ }^{\text {ème }}$ siècle) pour justifier un aspect fondamental: ces méthodes proposent un mouvement lent, souvent effectué allongé pour réduire l'effort, le plus petit possible. Cela n'est pas d'ailleurs sans poser problème: cet évitement du mouvement rapide, ce rejet de l'effort et de la douleur, n'excluent-ils pas a priori toute une série de mouvements, d'expériences vitales et de personnes? Toujours est-il que la référence à cette loi noue intimement deux aspects: les modalités de mouvement proposées et une certaine idée de la sensibilité et donc de l'attention recherchée. Il faut faire petit, doux et lent pour sentir le mieux possible toutes les moindres différences. Pour faire comprendre cette loi, Feldenkrais donne un exemple:

Par exemple, si je tiens un poids de 20 pounds, je ne peux pas percevoir si une mouche se pose dessus, parce que la plus petite différence de stimulus perceptible se situe entre $1 / 20$ et $1 / 40$, et qu'il faudrait donc ajouter ou enlever au moins un demi pound au poids porté pour que le changement devienne conscient [aware]. Si je tiens une plume, le poids d'une mouche peut faire une grande différence. [...] Une action de plus en plus fine n'est possible que si la sensibilité - c'est-à-dire, la capacité de sentir la différence - est améliorée ${ }^{7}$ (Feldenkrais, 2010b, p. 37).

La référence à la loi de Weber (1795-1878) et Fechner (18011887) est donc intrinsèquement solidaire de l'annonce implicite des objectifs de la méthode: effectuer un mouvement de plus en plus fin, pour développer une attention au changement de plus en plus sensible aux petites différences. En cherchant à affiner la sensation des petites différences plutôt qu'à entrainer musculairement à effectuer 
un certain vocabulaire de mouvements, la méthode Feldenkrais, et les méthodes somatiques en général, revendiquent un état de moindre effort en ces termes: réduire l'effort inutile dans le mouvement afin de développer une majeure sensibilité, et permettre ainsi une plus grande efficacité de mouvement. Ces notions d'inutilité et d'efficacité sont sans doute à interroger: utile à quoi ou pour quoi? Efficace dans quel sens? Autant de questions qui deviennent particulièrement urgentes à poser pour penser les usages contemporains de ces méthodes, au vu par exemple, de leur utilisation dans le cadre de pratiques de management en entreprise. Si le cadre de cet article ne nous permet pas de développer complètement cet aspect de la question, nous voulons tout de même rappeler que ce mouvement lent, petit, doux, est un paradigme fondamental qui s'appuie sur cette première référence à la psychophysique et son idée d'une sensibilité à la différence.

Les premières recherches psychophysiques se développent, avec Weber puis Fechner, à partir du projet visant à établir un rapport entre la force du stimulus et la puissance de la sensation, c'est-à-dire une corrélation entre des données alors considérées comme externes et physiques et d'autres comme internes et psychiques. Un projet qui alimentera les futurs chemins de la psychologie expérimentale mais aussi ceux des premières recherches de Freud, chemins sur lesquels nous ne nous aventurerons pas ici. Un projet qui vient en tout cas bouleverser, dès son origine, les idées de conscience de la philosophie, en concevant une sensibilité différentielle.

Contentons-nous donc ici de revenir sur cette référence à la loi de Weber-Fechner, pour comprendre les enjeux et les nouages conceptuels-pratiques d'une méthode qui viserait une attention dynamique, différentielle. Si la sensation devient un espace à part entière d'étude et de travail au cours du XIX ${ }^{\text {ème }}$ siècle, puis dans le $\mathrm{XX}^{\text {ème }}$ siècle, c'est que poussent au sein et aux abords de la philosophie des recherches qui prennent au sérieux un projet apparemment un peu fou: la réunion de considérations métaphysiques et d'une physiologie de la sensation. Cela commence avec le Weber de la loi de WeberFechner, au tout début du XIX ${ }^{\text {ème }}$ siècle, en Allemagne, qui conduit de longues expériences sur le toucher afin de rendre compte d'une relation entre stimulus et sensation. Que le toucher soit le premier des sens étudiés ainsi ne nous surprendra pas (Heller-Roazen, 2011), tant sa centralité est évidente. Thomas Ribot expliquait que Weber s'intéressait également à un problème historique: rendre compte de la 
sensation de soi, d'une sensation d'exister, parfois appelée cénesthésie. Il explique qu'Ernst Heinrich Weber «[...] entendait par ce mot: une sensibilité interne, un toucher intérieur qui fournit au sensorium des renseignements sur l'état mécanique et chimico-organique de la peau, des muqueuses et séreuses, des viscères, des muscles, des articulations» (Ribot, 1932, p. 22). Ce sera ce même Weber qui, le premier, énoncera la loi logarithmique du rapport entre stimulus et sensation, que Fechner reprendra et modifiera pour proposer ce qu' il nommera la loi Weber-Fechner.

L'idée pour nous n'est pas tant de chercher à savoir si cette loi se vérifie ou non - sachant que bien évidemment les recherches en la matière ont évolué depuis le début du XIX ${ }^{\text {ème }}$ siècle - mais de comprendre le substrat conceptuel et épistémologique qui entoure cette fameuse loi de Weber-Fechner, lorsque Feldenkrais et d'autres méthodes somatiques y font sans cesse référence pour justifier le mouvement lent et doux et le faire peu qui les caractérisent.

\section{Le Presque Pas et le À Peine}

Lorsque Fechner va s'attacher à l'étude de la sensation et aux conditions de sa mesure, il va d'abord déterminer deux seuils (threshold): la plus petite intensité en dessous de laquelle on ne sent rien - qu'il nomme perception «inconsciente» (Fechner, 1912) et qu'on peut comprendre comme la limite inférieure de la sensibilité - et la plus grande intensité possible, au-dessus de laquelle l'organe de sensation risque d'être endommagé. Dans l'intervalle entre ces deux seuils, Fechner établit une mesure basée non pas sur une valeur absolue de grandeur de la sensation selon la force d'un stimulus, mais sur la corrélation entre deux différences: celle entre deux intensités de stimuli et celle entre deux intensités de sensations.

Au moment où il cherche à établir une relation entre stimulus et sensation - pour ensuite traduire cela, telle est l'intention de Fechner, à la mesure de la "sensibilité interne» ou "conscience» (Fechner, 1912) - il se confronte, tout comme Weber avant lui, à l'impossibilité d'établir une unité de valeur pour mesurer la sensation comme on pourrait mesurer une étendue en millimètre ou un intervalle en seconde. La corrélation ne s'établit pas sur la grandeur d'un stimulus qui nous donnerait une valeur de base pour mesurer une sensation, mais sur un différentiel d'excitation qui provoque un passage de seuil sensible. Dans les termes de Fechner lui-même: 
La grandeur de la sensation $(\gamma)$ n'est pas proportionnelle à la valeur absolue du stimulus $(\beta)$, mais au logarithme de la grandeur du stimulus, quand ce dernier est exprimé dans le cadre de sa valeur de seuil (b), c'est-à-dire cette grandeur considérée comme l'unité où la sensation commence et disparaît. En résumé, elle est proportionnelle au logarithme de la valeur du stimulus fondamental ${ }^{8}$ (Fechner, 1912, p. 569).

Par exemple, la corrélation n'est pas directe entre la grandeur du poids déposé dans la main, la force exercée par mes muscles pour le porter, et l'intensité de la sensation que j'ai de ce poids; si corrélation il y a, c'est entre la différence du poids ajouté par rapport à celui déjà porté, et la sensation de changement d'intensité. La sensation est donc fondamentalement un passage: passage de l'inaperçu au perçu, un changement variant selon la tension préalable du muscle et la puissance d'augmentation du stimulus. Ce que l'on peut établir, c'est la plus petite différence de poids qui provoque une sensation, ou l'écart minimum pour que quelqu'un sente la différence, et non une mesure de la sensation en elle-même, qui ne saurait jamais avoir d'unité propre, absolue et universelle.

De là donc que la sensibilité est saisie comme un passage, et que la sensation elle-même peut être conçue comme passage de seuil:

Selon ce qui a été avancé jusque-là, notre formule de mesure correspond à l'expérience: 1 . Dans les cas d'égalité, où une différence de sensation reste la même lorsque l'intensité absolue du stimulus est altérée (loi de Weber). 2. Dans les cas des seuils, où la sensation elle-même cesse, et où son changement devient soit imperceptible soit à peine perceptible. Dans ce cas-là, lorsque la sensation atteint son seuil le plus bas; dans ce cas-ci, lorsqu'elle devient si forte qu'une augmentation de stimulus donnée est à peine remarquée. 3. Dans les cas opposés, entre les sensations qui s'élèvent en dessous du seuil de conscience, et celles qui ne l'atteignent pas - en résumé, les sensations conscientes et inconscientes $^{9}$ (Fechner, 1912, p. 572).

Passages de seuil et corrélation différentielle forment l'univers de pensée de Fechner. À la limite, toute sensation n'est qu'une dérivée du passage de seuil de sensibilité, entre imperceptible et perceptible, rejoué tout au long de la sensation et de ses différentes intensités. Sentir c'est sentir une différence, une différence comme passage, passage de seuil du presque rien et de l'à peine un peu (barely).

Reste que ce seuil sensible n'est pas exactement une ligne pure ou un instant t. Pour que le seuil soit sensible, il faut qu'il ait ce que nous appellerons une certaine épaisseur. Cette idée d'une philosophie 
de l'épaisseur apparaît dans l'essai que le philosophe William James consacre à Fechner (James, 1997, p. 77). Nous la reprenons ici pour penser la sensation différentielle: le seuil n'est sensible que dans son épaisseur singulière, dans le passage de sensibilité différentielle, qui prend un certain moment/trajet. De la sensation comme seuil, passage, délai minime, infime épaisseur, émerge une conception renouvelée de la conscience comme tendance, amorce d'un mouvement psychique dans une direction. C'est ainsi que James décrit la philosophie de Fechner:

[...] le (mouvement psychophysiques, tel que Fechner le nomme, est le terme le plus riche pour toute la réalité même. En tant que (mouvement) elle a une «direction; en tant que spsychique, la direction peut être sentie comme une 'tendances et comme tout ce qui se trouve relié aux tendances dans le sens d'une expérience intérieure - désir, effort, succès, par exemple; tandis qu'en tant que shysiques, la direction peut être définie en termes spatiaux et formulée mathématiquement ou sous la forme d'une ‘loi descriptive $^{10}$ (James, 1904, p. XIII-XIV).

De ce parcours dans les textes de Fechner, autour du projet psychophysique et de sa conception de la sensation et de la conscience, émerge l'idée d'une conscience comme passage de seuil, comme «vaguelettes» qui émergent à la surface (James, 1904, p. XIV-XV), comme tendance à l'à peine plus ou moins. Cet univers de pensée physique et métaphysique conjugue étonnamment le mesurable avec l'incommensurable, l'étendu avec l'inétendu, la quantité avec la qualité. Si le projet de la psychophysique interne rend compte de tendances plutôt que de choses ou de trajectoires accomplies, c'est que la conscience elle-même est passage, transition et tendance.

\section{Une Attention Tendancielle?}

En partant de l'attention dynamique et différentielle émergeant de l'expérience du scanning dans une séance de Feldenkrais et de son exposé dans ses textes théoriques, en passant par l'approfondissement de mention faite de la loi de Weber-Fechner et de son univers de référence, nous sommes amenés à effectuer un dernier pas: si l'on peut dire que la méthode Feldenkrais ne travaille pas à une prise de conscience d'un corps-objet mais à une attention au changement et aux petites différences, c'est dans la mesure où l'attention à travers le mouvement recherchée est bien plutôt une attention aux tendances à 
se mouvoir qu'une prise de conscience d'une partie du corps définie ou d'un mouvement en soi, comme globalité déjà effectuée.

C'est en tout cas ce que laissent penser les consignes d'une séance collective - tout à fait cadrées par ailleurs, et suivant à chaque leçon un déroulement très précis - lorsqu'elles invitent les élèves à imaginer le mouvement sans le faire, puis à porter leur attention sur la manière dont ils s'organisent juste avant d'effectuer le mouvement indiqué. Et alors ce serait ce passage de l'amorce à l'effectuation, l'épaisseur de cette tendance à se mouvoir, qui pourrait devenir le lieu-moment de l'apprentissage: un lieu-moment pour percevoir comment j'ai l'habitude de faire et laisser l'espace d'y insérer une nouvelle habitude en cours. Tel serait le sens de faire peu, toujours moins, d'à peine effectuer le geste, pour affiner l'attention au passage, au changement, au nouveau:

[...] le groupe est encouragé à plusieurs reprises à apprendre à faire un petit peu moins bien, autant que possible, en essayant fermement d'être moins rapide, moins vigoureux, mois gracieux etc. [...] On doit tout particulièrement mentionner les tout petits mouvements, presqu'imperceptibles, que j'utilise abondamment. Ils réduisent la contraction involontaire du muscle d'une manière étonnante ; en quelques minutes, en travaillant avec une jambe ou un bras par exemple, on peut la ou le sentir plus longue ou plus long, ou plus légère ou léger, que l'autre. Après le cours, les élèves continuent de sentir quelle est cette nouvelle manière d'agir $[. . .]^{11}$ (Feldenkrais, 2010a, p. 38).

L'idée de réduire l'effort, d'effectuer des mouvements petits et lents, d'abandonner la grâce et la vigueur, qui peut paraître étrange si l'on pense à un apprentissage du mouvement, a un objectif: affiner le seuil de son attention jusqu'à sentir l'à peine perceptible (barely perceptible)et ainsi clarifier la conscience, ou plutôt, à nouveau, l'attention (awareness) de l'épaisseur de ce seuil qu'est l'amorce du mouvement, le délai, moment-lieu, entre l'organisation de la posture juste avant de bouger et la tendance effectuant le mouvement:

Quand on a le choix, on peut effectuer l'action ou bien l'inhiber et annuler entièrement l'attitude préparatoire. Dans le groupe, nous cherchons à clarifier le délai entre l'attitude préliminaire pour l'action et son effectuation. Cette clarification ou attention améliore la fluidité et le contrôle volontaire des mouvements ${ }^{12}$ (Feldenkrais, 2010a, p. 39).

C'est donc, pour Feldenkrais, en exerçant son attention sur l'épaisseur du délai existant que l'on pourrait défaire et refaire dif- 
férentes manières d'agir, et non en exerçant une grammaire de mouvements tout faits. Cet apprentissage par l'attention à travers le mouvement ne cherche donc peut-être pas tant à avoir une représentation parfaite de tel ou tel mouvement, ou de telle ou telle partie du corps - sur le modèle d'une conscience comme représentation de quelque chose - qu'à développer une attention dynamique accompagnant les amorces du mouvement, les tendances sensibles et actives en cours.

En ce sens, l'affirmation initiale qui voulait que la méthode Feldenkrais, mais certainement aussi d'autres méthodes somatiques, ne cherchent pas tant à travailler sur le corps qu'à développer une attention à travers le mouvement, est solidaire d'une certaine conception de l'attention, sur laquelle elles s'appuient et qu'elles participent en même temps à produire une attention: a) différentielle (non seulement pour être attentif aux différences entre un côté et l'autre mais parce que toute sensation est elle-même différentielle, selon l'univers de pensée auquel la loi de Weber-Fechner fait référence); b) dynamique (pas une meilleure conscience de quelque chose, d'une image fixe d'un muscle ou d'un os par exemple, mais une attention au changement, nécessairement changeante elle-même, une attention à travers le mouvement nécessairement mouvante, relationnelle); et, finalement, c) tendancielle ( pas une conscience d'un mouvement, mais une attention aux manières d'entamer le mouvement, aux tendances en cours et aux directions esquissées).

Ainsi, en considérant la relation entre l'expérience de la méthode Feldenkrais, les textes de son fondateur, et les références conceptuelles qui les sous-tendent et qu'ils produisent, on peut voir émerger une attention, nourrie d'une sensation des toutes petites différences, intrinsèquement dynamique, qui porte moins sur un mouvement tout fait, ou sur le corps comme objet, que sur les tendances à se mouvoir et à sentir, les amorces du geste, les potentiels d'actions. Il faudrait alors voir dans quelle mesure s'ouvrirait ainsi la possibilité d'un apprentissage basé sur la variabilité des amorces et des esquisses, pariant sur la puissance d'effets d'une certaine épaisseur de l'attention, sur les transformations réelles d'une poétique à la fois virtuelle et effective du mouvement, et sur les redistributions concrètes et conceptuelles des lieux et des manières de sentir et de faire. 


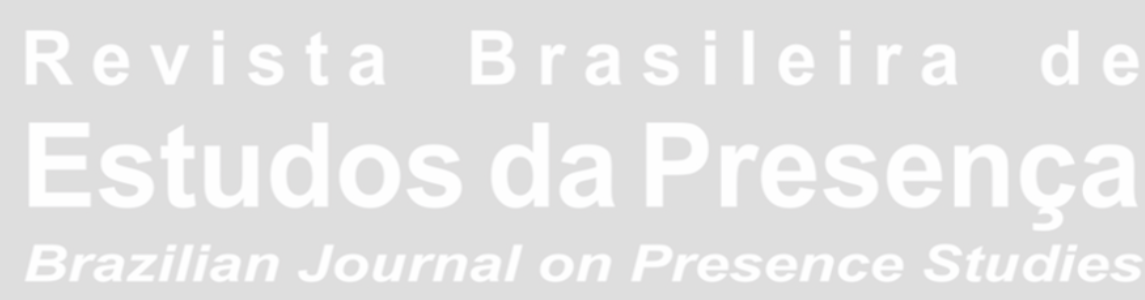

\section{Notes}

${ }^{1}$ Fondé par Marie Bardet, Carla Bottiglieri, Joanne Clavel, Isabelle Ginot, Beatriz Preciado, puis rejoint par Violeta Salvatierra. Pour plus d'information voir Soma\&Po. Somatiques, Esthétiques, Politiques (2014).

${ }^{2}$ Traduit de l'original en anglais: "[...] we must view human beings in their entirety. A person is made of three entities: the nervous system, which is the core; the body skeleton, viscera and muscles - which is the envelope; and the environment, which is space, gravitation, and society" (Feldenkrais, 2010a, p. 32).

${ }^{3}$ Traduit de l'original en anglais: "I begin by asking people to lie on their backs (after the same principle of reducing gravity) and learn to scan themselves. That is, they examine attentively the contact of their bodies with the flour and gradually learn to detect considerable differences - points where the contact is feeble or non-existent and others where it is full and distinct" (Feldenkrais, 2010b, p. 76).

${ }^{4}$ Traduit de l'original en anglais: "The mental scanning consists of listening and becoming aware of the difference of sensation in the motor memory of the muscles of the two halves, and the sensation of change of orientation in space" (Feldenkrais, 2010a, p. 39).

${ }^{5}$ C'est Feldenkrais qui souligne: "[...] sensitivity - that is the ability to feel the difference [...]" (Feldenkrais, 2010a, p. 37).

${ }^{6}$ Traduit de l'original en anglais: "The gradual reduction of useless effort is necessary in order to increase kinesthesic sensitivity, without which a person cannot become selfregulating. The Fechner-Weber law shows clearly that this is so. This law states that for a wide range of human sensation and activity, the difference in stimulus that produces the least detectable difference in sensation is always the same ratio to the whole stimulus" (Feldenkrais, 2010b, p. 37).

${ }^{7}$ Traduit de l'original en anglais: "For example, if I hold a 20 pound weigh, I cannot detect a fly sitting on it because the least detectable difference of stimulus is from $1 / 20$ to $1 / 40$ and therefore at least half a pound must be added or subtracted from the carried weight to become aware of the change. If I old a feather, the weight of a fly makes a great difference. [...] Finer and finer performance is possible only if the sensitivity - that is, the ability to feel the difference - is improved" (Feldenkrais, 2010b, p. 37).

${ }^{8}$ Traduit de l'original en anglais: "The magnitude of the sensation $(\gamma)$ is not proportional to the absolute value of the stimulus ( $\beta$ ), but rather to the logarithm of the magnitude of the stimulus, when this last is expressed in terms of its threshold value (b), i.e. that magnitude considered as unit at which the sensation begins and disappears. In short, it is proportional to the logarithm of the fundamental stimulus value" (Fechner, 1912, p. 569).

${ }^{9}$ Traduit de l'original en anglais: "According to the foregoing our measurement formula corresponds to experience:1. In the cases of equality, where a sensation difference remains the same when the absolute intensity of the stimulus is altered (Weber's law); 2. In the cases of the thresholds, where the sensation itself ceases, and where its change becomes either imperceptible or barely perceptible. In the former case, when the sensation reaches 


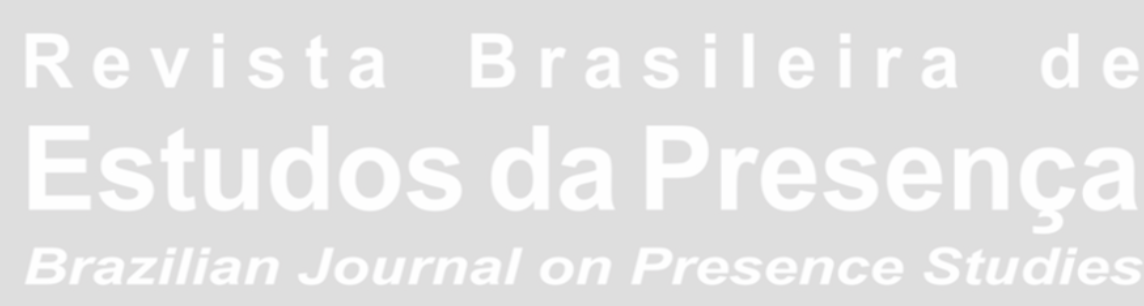

ISSN 2237-2660

its lower threshold; in the latter case, when it becomes so great that a given stimulus increase is barely noticed; 3 . In the contrasting cases, between sensations which rise above the threshold of consciousness and those that do not reach it, -- in short, conscious and unconscious sensations" (Fechner, 1912, p. 572).

${ }^{10}$ Traduit de l'original en anglais: "[...] psychophysical movement, as Fechner calls it, is the most pregnant name for all the reality that is. As movement it has a direction; as psychical the direction can be felt as a tendency and as all that lies connected in the way of inner experience with tendencies, - desire, effort, success, for example; while as physical the direction can be defined in spatial terms and formulated mathematically or otherwise in the shape of a descriptive law" (James, 1904, p. XIII-XIV).

${ }^{11}$ Traduit de l'original en anglais: "[...] the group is repeatedly encouraged to learn to do a little less well than is possible when trying hard to be less fast, less vigorous, less graceful, etc. [...] Special mention must be made of very small, barely imperceptible movements that I use extensively. They reduce involuntary contraction in the muscle in an astonishing way; in a few minutes by working on one leg or arm, for example, it may be made to feel longer and lighter than the other. After the lesson the pupils keep on feeling what the new way of action is" (Feldenkrais, 2010a, p. 38).

12 Traduit de l'original en anglais: "When there is a choice, we can complete the action or else prevent it and cancel the preparatory attitude entirely. In the group, we work on clarifying the delay between the preliminary attitude for action and its completion. This clarification or awareness improves the fluency and voluntary control of movements" (Feldenkrais, 2010a, p. 39).

\section{Références}

BARDET, Marie; GINOT, Isabelle. Habit and Change: discovering the present. An essay on the invention of time in Feldenkrais method: learning through movement, questions of temporality. Writings on Dance, Melbourne, Writing on Dance Inc., n. 25, p. 10-27, summer 2012.

FECHNER, Gustav Theodor. Elements of Psychophysics. Dans: RAND, Benjamin (Dir.). The Classical Psychologists: selections illustrating psychology from Anaxagoras to Wundt. Boston: Houghton, Mifflin and Company, 1912. P. 562-572.

FELDENKRAIS, Moshe. Mind and Body. Dans: BERINGER, Elizabeth (Dir.). Embodied Wisdom. California: North Atlantic Books, 2010a. P. 27-44.

FELDENKRAIS, Moshe. Man and the World. Dans: BERINGER, Elizabeth (Dir.). Embodied Wisdom. California: North Atlantic Books, 2010b. P. 59-68.

HANNA, Thomas. What is Somatics. Dans: JOHNSON, Don Hanlon (Dir.). Bone, Breath \& Gesture. Berkeley: North Atlantic Books, 1995. P. 341-352.

HELLER-ROAZEN, Daniel. Une Archéologie du Toucher. Paris: Le Seuil, 2011.

JAMES, William. Introduction. Dans: FECHNER, Gustav Theodor. The Little Book of Life after Death. Boston: Little, Brown \& Company, 1904. P. VII-XIX. 


\section{Revista Brasithira de \\ Estudos da Presenca

ISSN 2237-2660

JAMES, William. Fechner. In: FECHNER, Gustav Theodor. Anatomie Comparée des Anges. Paris: Éditions de l'Éclat, 1997. P. 67-99.

RIBOT, Thomas. Les Maladies de la Personnalité. Paris: Alcan, 1932.

SOMA\&PO. Groupe de Recherches "Soma \& Po: somatiques, esthétiques, politique". Paris 8 Danse, Saint-Denis, [n.d.]. Disponible sur: <http://www.danse.univ-paris8.fr/ recherche_en_cours.php?pt_id=7>. Consulté le: 13 août 2014.

Marie Bardet est docteure en esthétique et philosophie contemporaines. Elle est actuellement chercheuse associée à Soma\&Po au Département de Danse de l'Université Paris 8. Auteure de Penser et Mouvoir (Harmattan, Paris, 2011 ; Cactus, Buenos Aires, 2012), fait partie de la commission du doctorat en Art de l'Institut Universitaire des Arts à Buenos Aires.

E-mail: bardetmarie@hotmail.com

Ce texte inédit, révisé par Annelyse Gayraud, est également publié en portugais dans ce numéro.

Reçu le 30 juillet 2014 Accepté le 02 septembre 2014 